\title{
OSTEOMYELITIS IN THE NEWBORN
}

\author{
BY \\ JOHN THOMSON and IAN C. LEWIS \\ From the Department of Child Life and Health, University of Edinburgh \\ (Received for Publication February 6, 1950)
}

The earlier literature on osteomyelitis of very young infants dealt largely with osteomyelitis of the jaw and its probable relationship to mastitis in the nursing mother (Marx, 1920; Bass, 1928; Karplus, 1928; Wilensky, 1932; Poncher and Blayney, 1934).

Metastatic osteomyelitis due to umbilical sepsis was mentioned by Fraser (1924) who collected some 30 cases over a period of four years. He said that a fatal result was almost inevitable. Baumgartner (1920) recorded a case of osteomyelitis of the skull, Madier (1922) one of osteomyelitis of a vertebra, and Palew (1931) one of gonoccoccal osteomyelitis.

In a study of septicaemia of the newborn infant Dunham (1933) found that osteomyelitis occurred in $10.3 \%$ of the cases and that three out of four such cases died. Mount (1935) reported a fatal case of septicaemia with osteomyelitis of the humerus. Green (1935) recorded a case of osteomyelitis of the humerus, with recovery, where the original lesion may have been a furuncle of the chin at the age of two weeks. The same author with Shannon (1936) published a series of 23 cases of osteomyelitis which occurred before the age of six months. The mortality rate was $45 \%$. They related extra-osseous infection and trauma to the osteomyelitis and stressed skin infection and omphalitis as primary lesions. They considered that osteomyelitis was not a rare disease in infants and emphasized that the treatment was that of the general condition rather than of the local one.

The poor prognosis given by Fraser and Dunham was not confirmed by Dillehunt (1935) among whose cases there were two in the neonatal period. He said:

\footnotetext{
- The disease is rare in infants. It is relatively benign, and neglect to treat it in infants is less harmful than in adults. The prognosis is favourable. Sequestra do not form. Spontaneous resolution without treatment may occur. Foci of infection may become sterile.'
}

In the three cases recorded by Cass (1940) there was relatively little systemic disturbance though in two of them there was pyrexia. Apart from deformity, the prognosis was stated to be good in the absence of infection of the lungs, peritoneum, meninges or skin. Four cases of osteomyelitis all of which recovered were recorded by Stone (1942). He stated that the course of the disease in the newborn was benign and that the treatment of the osseous lesion was of secondary importance. He gave a good prognosis for life and function.

The benign course of the disease was also mentioned by Einstein and Thomas (1946) who said that involvement of a joint was common and was the most serious complication. Of their ten cases under the age of six months one was in the neonatal period. A case of osteomyelitis with recovery in a 19-day old infant was reported by Shulman (1946).

The confusion of opinion in the prognosis of osteomyelitis was in large measure clarified by Greengard (1946). He recorded ten cases of acute haematogenous osteomyelitis in infants, one of which was in the neonatal period. He suggested that in the newborn infant there were two forms of this disease; first, a 'benign' form in which there was little or no complaint other than local disability and no history of preceding illness, and second, a severe form in which there was systemic evidence of a violent infection and the local disease made its appearance as a complication. In this latter group the mortality was high. Hutter (1948), recording three cases of neonatal osteomyelitis, agreed with Greengard's classification.

Because of the number of cases of osteomyelitis in the neonatal period which have been recorded it may be said that this disease should no longer be considered a rare one: yet it has seldom been reported from a maternity hospital. The four cases now recorded were prematurely born infants who from birth were under the daily care and supervision of a paediatrician. Thus the earliest clinical manifestations of morbidity were observed. 


\section{Case Reports}

Case No. 1. This immature male infant, the result of a ninth pregnancy, was born spontaneously of unrelated parents on July 5, 1943. He weighed $5 \mathrm{lb} .7 \mathrm{oz}$. at birth. Progress was normal until the ninth day when onychia of the right middle finger was observed. Despite local treatment with gentian violet and fomentations, and chemotherapy with sulphathiazole g. $\frac{1}{1}$ four-hourly, the inflammation spread. The finger, and finally the whole hand, was involved. A discharge of thin pus came to the surface over the dorsal aspect of the distal joint of the finger which was incised. The pus was not investigated bacteriologically. The sinus was healed four weeks from the appearance of the onychia, when all swelling had disappeared except that affecting the finger. At this stage the finger was examined radiologically (Fig. 1). Almost complete destruction of the bone of

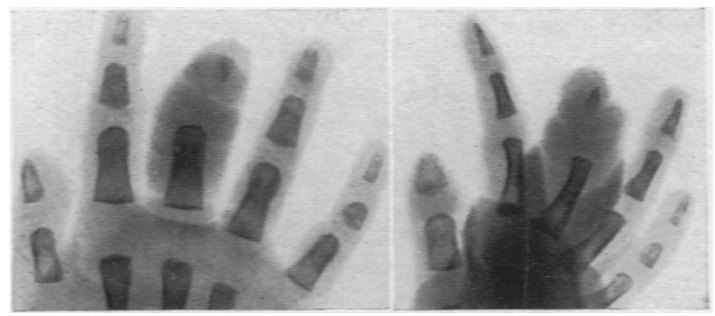

FIG. 1.-Radiographs showing pinpoint of bone in the middle phalanx and considerable destruction of proximal portion of distal phalanx.

the middle phalanx and most of the proximal part of the distal phalanx was seen.

The infant was discharged aged 45 days. He weighed $6 \mathrm{lb} .3 \frac{1}{2} \mathrm{oz}$. No systemic disturbance occurred during this acute illness (Fig. 2).

The infant has been observed at intervals since discharge from hospital. A radiograph shows the extent of bone recovery at the age of 5 years and 7 months (Fig. 3).

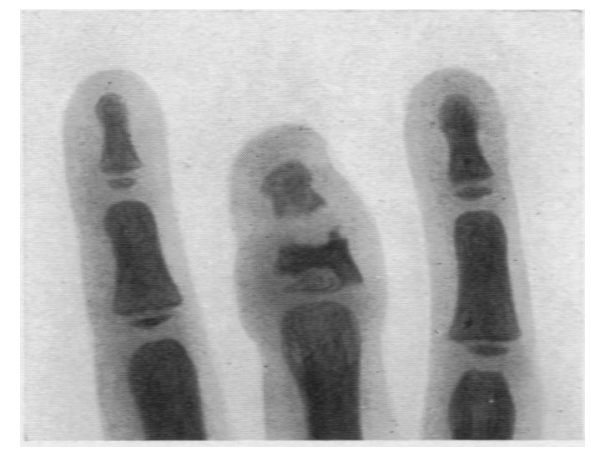

FIG. 3.-Radiograph showing extent of bone recovery.

Case No. 2. This female infant was born spontaneously on January 13, 1943. She weighed just over $5 \mathrm{lb}$. At 12 days staphylococcal pustules were seen in the right axilla and at the right elbow. They were treated with a $1_{0}$ aqueous solution of gentian violet. From the pustule at the elbow inflammation spread rapidly into the soft tissues. A fluctuating swelling near the proximal end of the ulna and on the dorsal aspect was incised on the eighteenth day. From the pus thus obtained Staphylococcus aureus was grown. A diagnosis of periostitis or osteomyelitis was made three days later, but was not confirmed on radiological examination. During the period of clinical inflammation there was virtually no general systemic disturbance (Fig. 4). When 54 days old a second radiograph of the elbow joint region confirmed the original diagnosis of osteomyelitis (Fig. 5).

The infant made good progress and an uneventful recovery. Excellent functional use of the arm has been obtained, so that at 6 years old pronation and supination were complete. The only disability was a limitation of flexion at the elbow (Fig. 6).

Case No. 3. Born on January 30, 1947, this spontaneously delivered immature male infant weighed $3 \mathrm{lb} .2 \frac{1}{2}$ in. There was slight oedema of the legs, but the general colour was good and breathing was

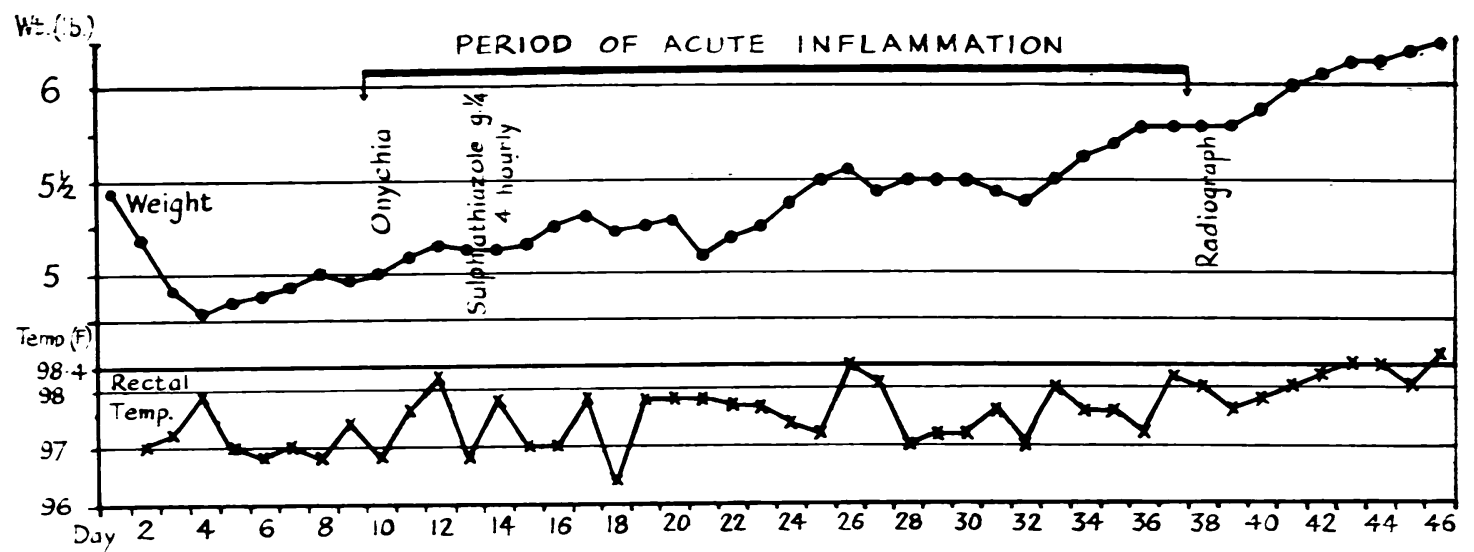

FIG. 2.-Chart of Case 1 showing absence of systemic disturbance during period of acute inflammation. 


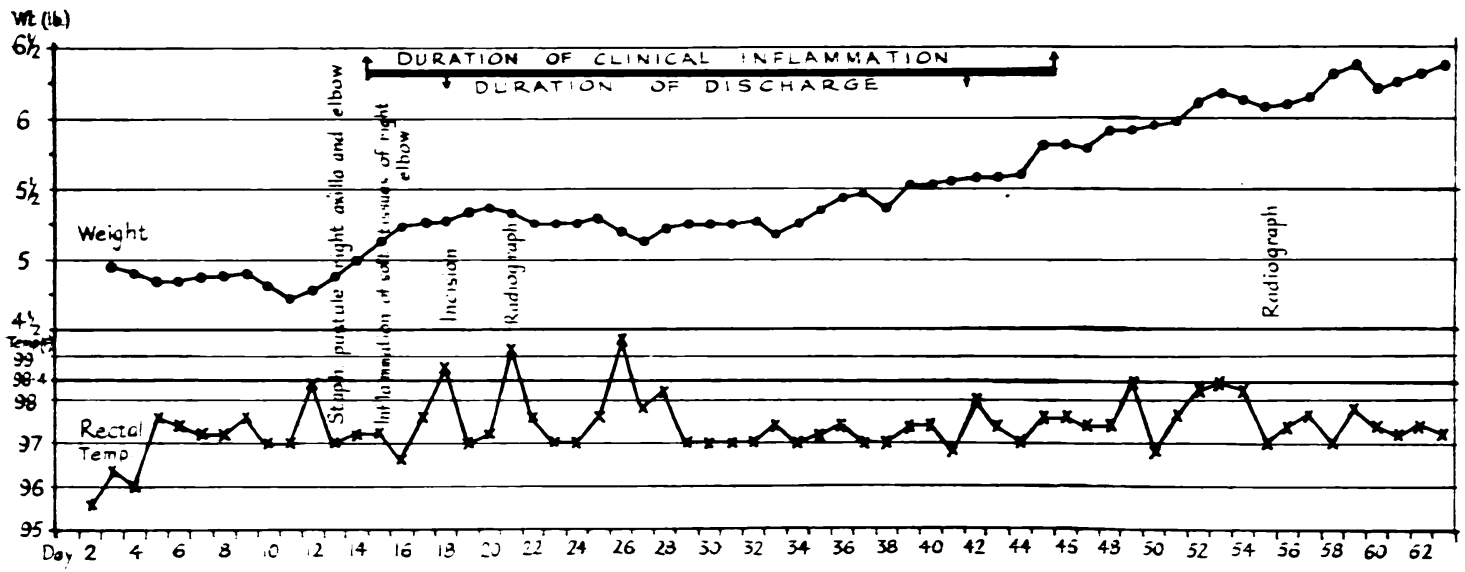

FIG. 4. - Chart showing only slight halt in weight gain and virtual absence of pyrexia. There was no general systemic disturbance.

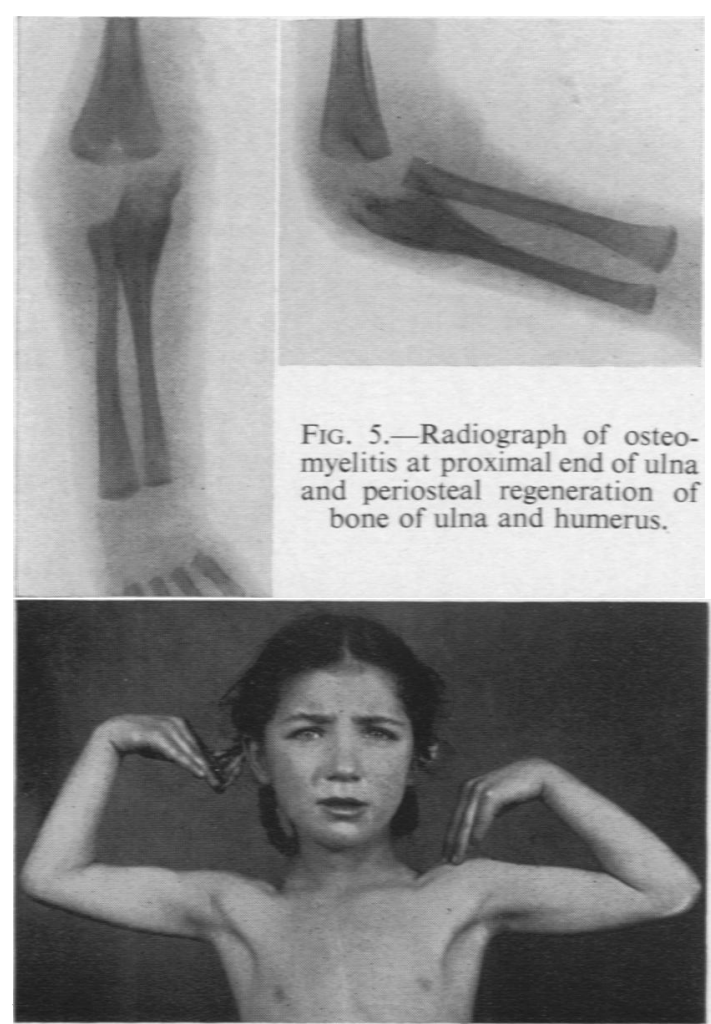

Fig. 6.-Limitation of flexion at elbow.

satisfactory. Because there was gastro-enteritis in the unit when the infant was born he was given sulphaguanidine as a prophylactic measure. The infant was fed by gavage and made satisfactory progress. On the night of the thirteenth day respirations became rapid and shallow and there was some vomiting. Though on clinical examination no other abnormality was discovered, a respiratory infection was presumed and penicillin and sulphathiazole were exhibited from the fourteenth day onwards (Fig. 7). Crepitations were heard in the right lung on the following day and x-ray examination suggested bronchopneumonia.

Swelling over the anterior and superior aspects of the left shoulder was seen on the sixteenth day. On aspiration, blood-stained pus was obtained and from it Staphylococcus pyogenes albus was grown. It was insensitive to penicillin. An identical organism was cultured from the blood on the twentieth day. On the same day small septic spots appeared on both arms and became haemorrhagic. The presence of osteomvelitis of the head of the left humerus was chown radiologically on the twenty-second day (Fig. 8). At the same time a localized inflammatory reaction developed in the left ankle and four days later the proximal end of the left tibia was involved. Osteomyelitis of the tibia was demonstrated radiologically (Fig. 9), but the same clinical diagnosis for the ankle was not confirmed. The shoulder region, which had been aspirated three times, was opened and drained on the thirty-first day, when the ankle was similarly dealt with. On the following day loose green stools appeared and sulphaguanidine medication was begun, but stopped in four days when these signs had disappeared. There was now little discharge from the shoulder and none from the ankle. A respiratory infection was still manifest in a nasal discharge. This became more profuse on the day of death when crepitations were again heard in the right lung. A severe cyanotic attack occurred and when this recurred the infant died, on the thirty-ninth day.

Necropsy and histological examination were performed. The findings were as follows:

RESPIRATORY SYSTEM. A resolving pneumonia of haematogenous origin, and minute, apparently quiescent, pyaemic abscesses in both lungs.

Locomotor SYSTEM. Osteomyelitis of the head and neck of left humerus. Left shoulder joint intact, but distended with pus. Osteomyelitis of the proximal end 


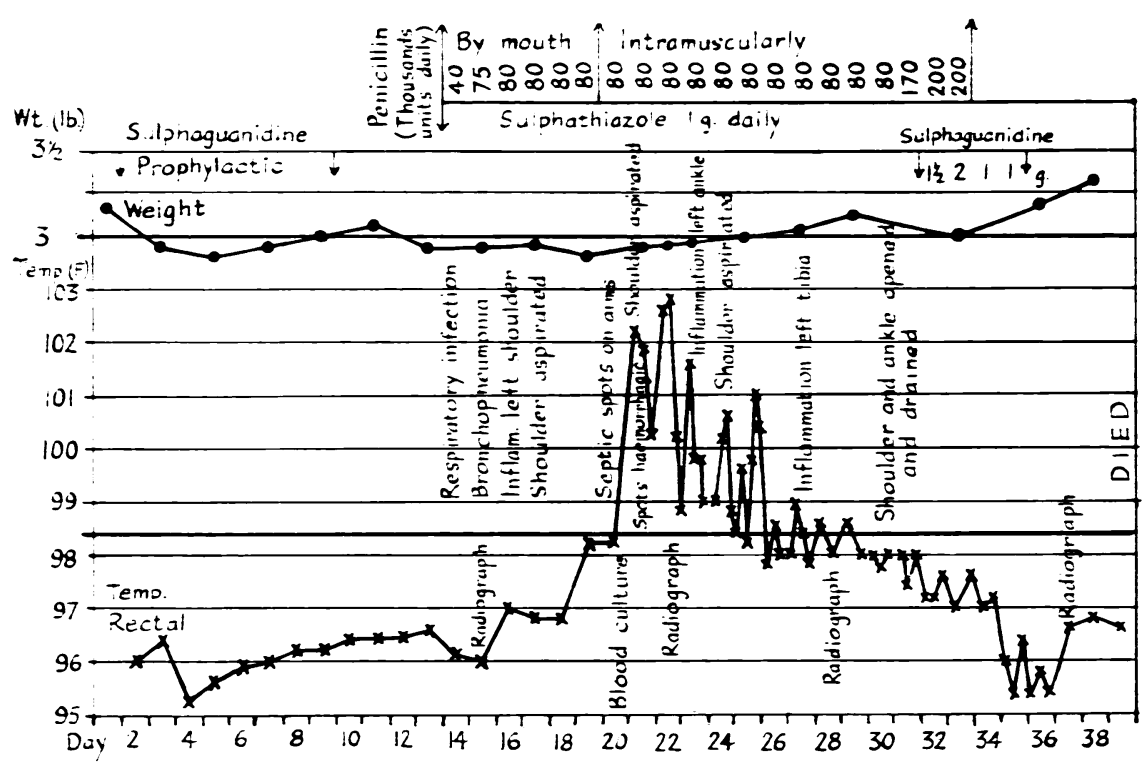

FIG. 7.-Chart showing general systemic disturbance. of the left tibia. Purulent arthritis of the left ankle joint and left tarsal joints.

Case No. 4. This male infant was spontaneously delivered on November 11, 1948. He weighed $5 \mathrm{lb} .2 \frac{3}{4} \mathrm{oz}$.

Progress was satisfactory until the fifteenth day when irritability and anorexia were observed. On clinical examination only mild paronychia of the left fifth toe was observed. Oral penicillin, 30,000 units fourhourly, was prescribed. Pyrexia and loss of weight occurred in the next 24 hours (Fig. 10). The infant appeared grey and toxic. The eyes were sunken. There was localized inflammation over the right medial malleolus, and movement of the ankle caused the infant to cry. On the third day of the illness pus was aspirated from the ankle and a culture of penicillin-resistant Staphylococcus aureus was obtained. At the same time the left wrist was inflamed. Oral penicillin was changed to intramuscular injections of 100,000 units three-hourly. On the fifth day of illness a swelling on the anterolateral aspect of the upper third of the right thigh was aspirated and thick pus obtained. By this time the inflammation of the left wrist subsided, but the right ankle remained swollen and fluctuating. Oral penicillin, 200,000 units threehourly, was given again. Some clinical improvement in the child's condition was apparent at this time, and despite the persistence of pyrexia the improvement was maintained. He lost the grey appearance of a desperately ill infant. On the ninth day the localized swelling at the right ankle was incised, and from the pus a culture of Staphylococcus aureus, resistant to over 100 units of penicillin per $\mathrm{ml}$. but sensitive to 2 units of streptomycin per $\mathrm{ml}$., was obtained. The discharge from the ankle was persistent. A probe could be passed into the ankle joint.

$X$-ray examination on the fifteenth day showed destruction and dislocation of the head of the right femur (Fig. 11). An unsuspected lesion of the fourth right costochondral joint was also disclosed. The right leg was kept abducted and externally rotated with a Putti mattress splint. On the nineteenth day of the illness streptomycin medication was begun, and $100 \mathrm{mg}$. were given daily for nine days. All inflammation subsided rapidly and the infant made good clinical progress. A radiograph on the fortieth day of illness showed regeneration of bone (Fig. 12). The infant was discharged when 93 days old, and weighed $8 \mathrm{lb}$. $7 \frac{1}{2} \mathrm{oz}$.

Fig. 8.-Osteomyelitis head of left humerus. 


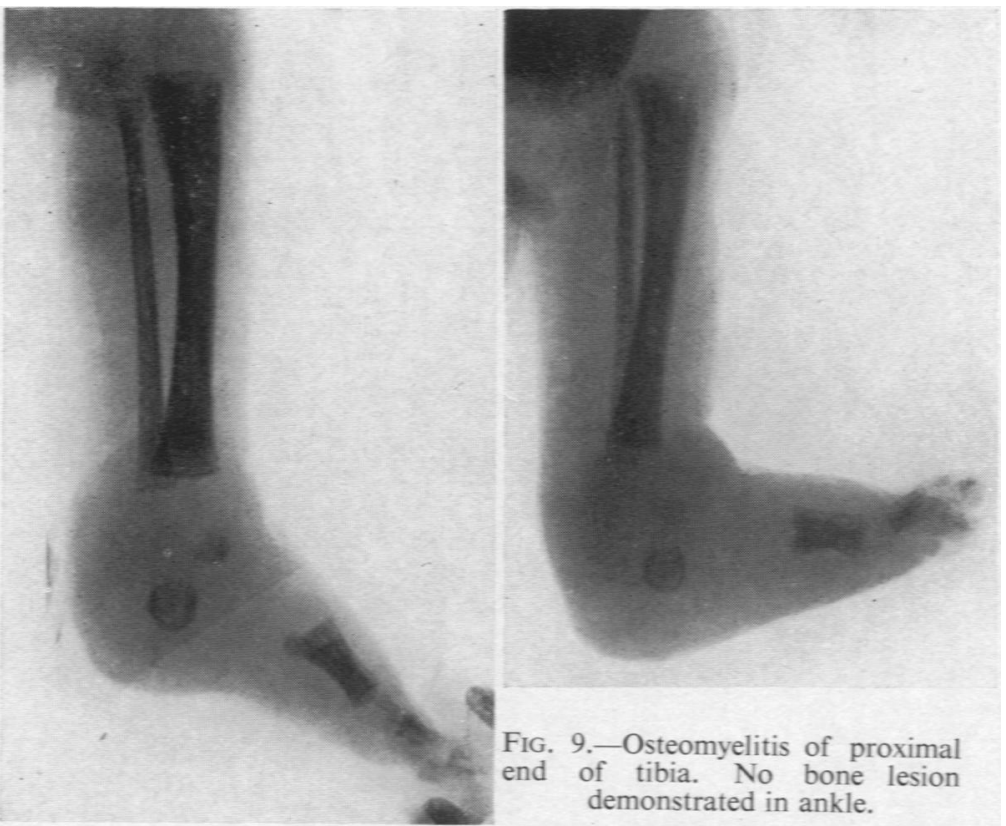

disease of the fourth right costochondral joint. There was complete destruction of the head of the right femur, some malformation of the proximal end of the shaft, and some upward displacement. The acetabulum was healthy (Fig. 13).

\section{Discussion}

The consensus of opinion concerning the aetiology of neonatal osteomyelitis is in favour of an antecedent infection. This may be omphalitis, as emphasized by Fraser, or a respiratory infection as in Case No. 3, or, most commonly, a skin lesion. Skin lesions are very prevalent in the newborn period and are invariably staphylococcal in type. Over a three-year period in a maternity hospital Henderson (1943) recorded 550 cases, an

The child's progress has been supervised at the infant welfare clinic during the first year of life, and he has also attended the orthopaedic department. There have been no illnesses. At one year old he weighed $19 \mathrm{lb} .4 \mathrm{oz}$, had five teeth, and was an alert, happy child who on the slightest provocation exhibited a strong desire to stand on his feet.

Radiological examination at this age showed that the right ankle joint had healed completely and had about 20 degrees of movement. There was no evidence of incidence of $6 \cdot 8^{\circ}{ }_{\mathrm{o}}$. He reported no complications other than conjunctivitis, and there were no deaths. These lesions are usually small, few in number, and transient. The relative infrequency with which complications appear, and the ease with which the majority of these lesions yield to simple therapeutic measures, largely obscures the potential danger which exists in all such cases.

It is significant that all our four cases were

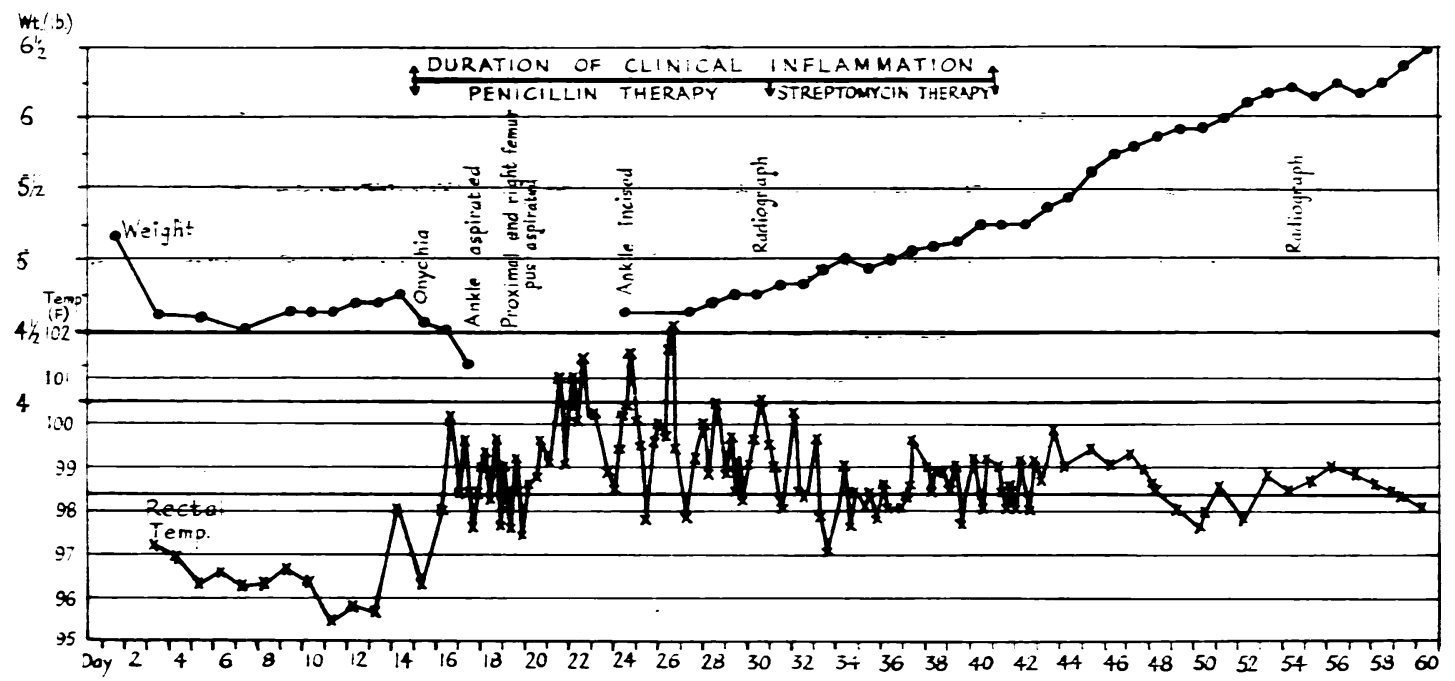

FIG. 10.-Clinical chart showing grossly abnormal temperature and weight loss at beginning of illness. 


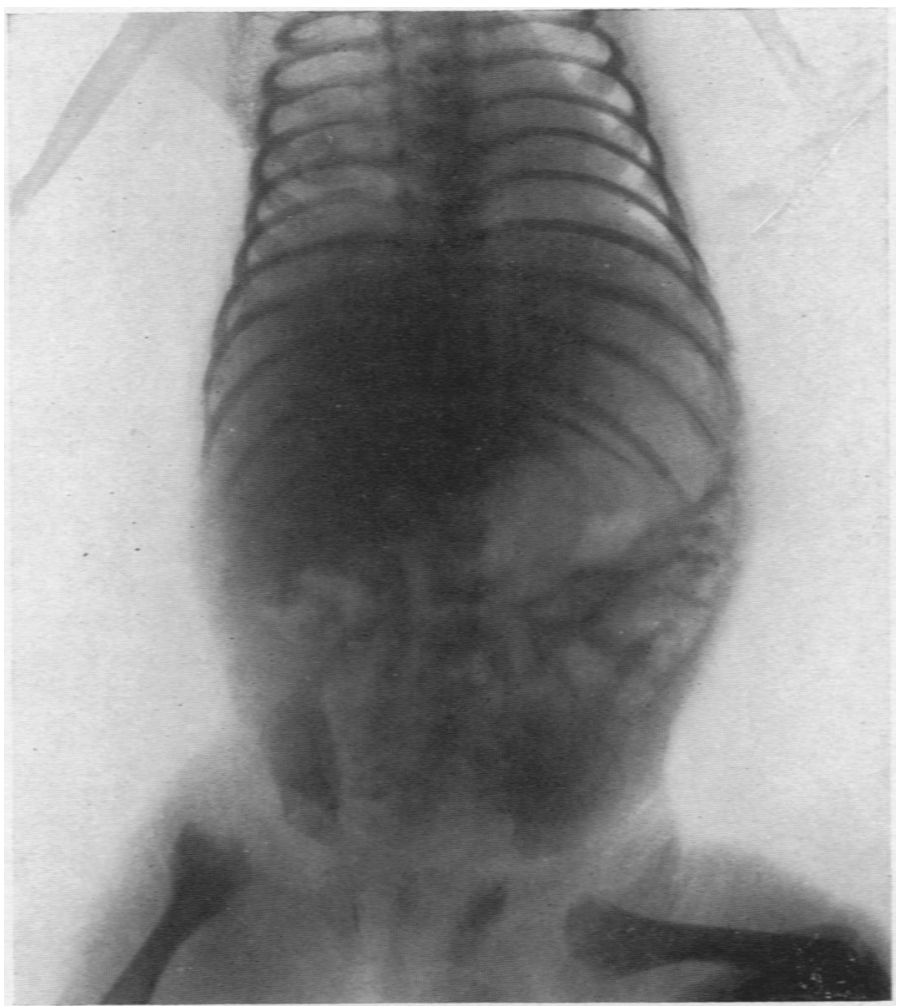

Frg. 11.-Radiograph illustrating rib and hip joint lesions.

immature infants. Such infants are immunologically immature, and not only much more susceptible to bacterial invasion than full-term infants, but also much less able to combat an established infection.

These four cases are divisible into two clinical groups. Cases 1 and 2 showed no systemic

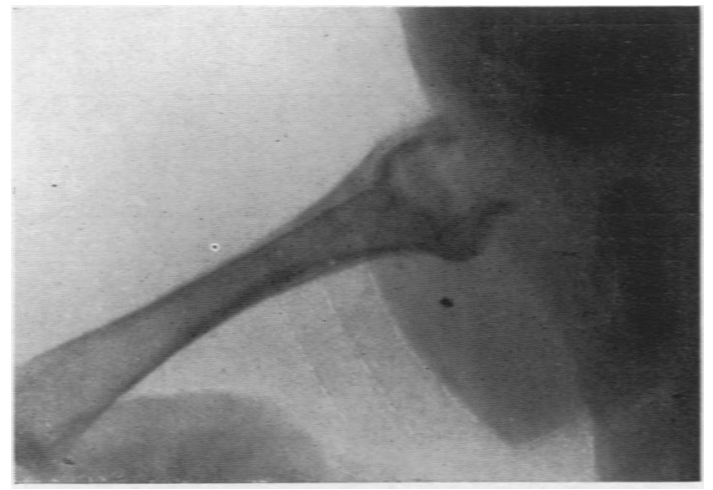

Fig. 12.-Radiograph illustrating regeneration of bone on the fortieth day of the illness. disturbance and ran a relatively mild or 'benign' course, whereas Cases 3 and 4 showed a gross systemic disturbance which ended fatally for one of them. This division of cases tends to confirm Greengard's clinical classification (1946), which is supported by Hutter (1948).

It can hardly be doubted that in Cases 3 and 4 the multiple osteomyelitis was a complication arising from a blood-borne infection. This may have been of respiratory origin in Case 3, and have arisen from skin infection in Case 4.

In Cases 1 and 2 a feature was the intimate relationship of the site of the osteomyelitis to the site of the initial inflammatory lesion in the soft tissues. The circumscribed nature of the lesion throughout the illness and the absence of any gross systemic disturbance might have suggested that one was dealing with a direct extension of a local inflammatory process. From this point of view it is unfortunate that no blood cultures were attempted in Cases 1 and 2. Moreover, any consideration of such a suggestion must be tempered by the knowledge that Hutter obtained positive blood cultures from a case of 'benign' neonatal osteomyelitis.

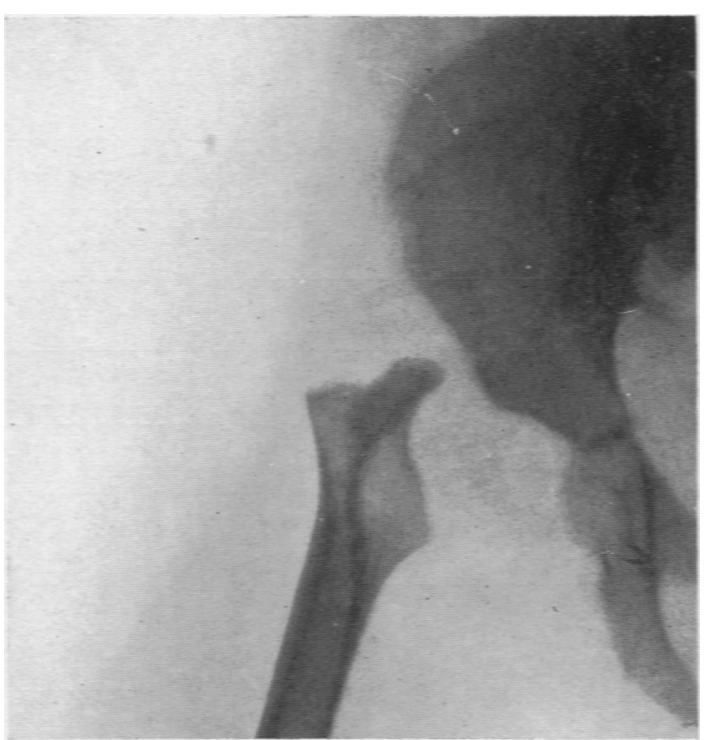

Fig. 13.-Radiograph showing condition of right hip joint at one year after illness. 
The three surviving cases each show some degree of permanent disablement. The extent of the disability in Cases 1 and 2 is slight and the functional result is very good. These two cases resemble those described by Cass (1940), who pointed out the risk of deformity in mild cases, and those described by Einstein and Thomas (1946) who said that involvement of a joint was a common and serious complication in "benign " cases.

The degree of loss of function sustained from neonatal osteomyelitis is not determined by the - benignity ${ }^{\circ}$ or otherwise of the course of the disease in the acute phase, but rather by the extent to which natural healing takes place and by the functional nature of the affected bone and joint. This is illustrated in Case 4 which shows the greatest degree of permanent disability of the three surviving cases. The much greater degree of disablement is caused by the destruction of a joint which should provide a high degree of stability. It is not possible in this case to assess the ultimate extent of the functional disability. It is hoped that much may be accomplished by orthopaedic means.

Because of the residual disability so often present in 'benign' or mild cases, one feels that the term - benign is a misnomer whose use should be discontinued. With this suggestion Greengard and Hutter are in agreement.

The high mortality rate hitherto recorded in neonatal osteomyelitis is not likely to continue, for the advent of chemotherapy and antibiotic treatment is exerting an ameliorating influence on the prognosis of this disease. This is well illustrated by Hutter, who records that during 1934-43 in the Children's Hospital, Los Angeles, there were 12 cases of haemolytic staphylococcal osteomyelitis in children under the age of six months. The mortality rate was $58^{\circ}{ }_{\mathrm{o}}$. In the subsequent period from 1943-47 there were six similar cases in the same age period. They were all treated with penicillin and chemotherapy, with no deaths.

The increase in the survival rate may result in the sequelae of neonatal osteomyelitis being seen more frequently in orthopaedic hospitals and clinics.
This premise cannot be based on the small number of cases now recorded, but the two groups mentioned by Hutter offer highly suggestive evidence. In the 1934-43 groups none of the survivors showed residual deformity, but in the 1943-47 groups two-thirds of the survivors showed residual deformity involving one or more joints.

\section{Summary}

Four cases of neonatal osteomyelitis occurring in immature infants are recorded.

They are divisible into two clinical groups: one relatively mild, the other severe and carrying considerable risk to life.

The potential danger existing from staphylococcal skin lesions in the neonatal period is stressed and the particular susceptibility of the immature infant.

Attention is drawn to a probable increase in the number of cases of disability following neonatal osteomyelitis which may be seen in orthopaedic clinics.

The adjective term 'benign" which has been applied to the mild group of cases is a misnomer.

\section{REFERENCES}

Bass, M. H. (1928). Amer. J. Dis. Child., 35, 65. Baumgartner, J. (1920). Rev. med. Suisse rom., 40, 816. Cass, J. M. (1940). Arch. Dis. Childh., 15, 55.

Dillehunt, R. B. (1935). Surg. Gynec. Obstet., 61, 96.

Dunham, E. C. (1933). Amer. J. Dis. Child., 45, 229.

Einstein, R. A. J., and Thomas, C. G. (1946). Amer. J. Roentgenol., 55, 299.

Fraser, J. (1924). Brit. med. J., 2, 605.

Green, W. T. (1935). J. Amer. med. Ass., 105, 1835.

- , and Shannon, J. G. (1936). Arch. Surg., Chicago, 32, 462.

Greengard, J. (1946). Med. Clin. N. Amer., 30, 135.

Henderson, J. L. (1943). Edinb. med. J., 50, 535.

Hutter, C. G. (1948). J. Pediat., 32, 522.

Karplus, D. (1928). Z. Kinderheilk, 45, 732.

Madier, J. (1922). Nourrison, 10, 168.

Marx, E. (1920). Nederl. Tijdschr. Geneesk., 2, 294.

Mount, W. B. (1935). Amer. J. Obstet. Gynec., 29, 126.

Palew, P. (1931). Amer. J. Surg., 13, 246.

Poncher, H. G., and Blayney, J. R. (1934). Amer. J. Dis. Child., 48, 730 .

Shulman, B. H. (1946). J. Amer. med. Ass., 130, 854.

Stone, S. (1942). Amer. J. Dis. Child., 64, 680.

Wilensky, A. O. (1932). Ibid., 43, 431. 